\title{
LOS ESCRITOS DE GEORG SIMMEL
}

\section{David Lazcano y Yolanda Mutiloa}

La primera recopilación bibliográfica de la obra de G. Simmel la realiza Nicholas J. Spykman en su libro The Social Theory of G. Simmel, publicado en 1925. Posteriormente, Erich Rosenthal y Kurt Oberlaender, en su ensayo «Books, Pappers and Essays by Georg Simmel», The American Journal of Socio$\operatorname{logy}$, vol. 51, 3, 1945, realizan una ampliación de la literatura sobre Simmel, que queda recogida en el apéndice bibliográfico que confeccionan Kurt Gassen y Michael Landmann en el libro Buch des Dankes and Georg Simmel. Briefe, Erinnerungen, Bibliographie, publicado en 1958. Estos dos compiladores realizan la agrupación de toda la literatura de y sobre Simmel con el mayor desglose y detalle posibles, aprovechando el aniversario del nacimiento de Simmel. Nosotros hemos tenido en cuenta todo este legado bibliográfico y hemos incluido la literatura sociológica sobre Simmel producida desde 1958. Esto lo hemos hecho de la siguiente manera: en primer lugar, incluimos una lista de los libros escritos por Simmel según su fecha de publicación original; en segundo lugar, incluimos una lista de los artículos publicados según su fecha original de publicación, por la razón de que se han realizado traducciones de estos artículos a diversos idiomas que contienen compilaciones distintas; en tercer lugar, incluimos una lista de las principales compilaciones de artículos; en cuarto lugar, incluimos una lista de las Obras Completas, cuya edición ha comenzado Ottheim Ramstedt y sus discípulos (de estas obras completas, de 24 volúmenes, han aparecido los siguientes: 2,3 , 4, 5, 6, 7, 8, 9, 10, 11 y 14);

\section{Reis}


en quinto lugar, incluimos una lista de las traducciones de sus obras realizadas al español, al francés, al inglés y al italiano; finalmente, incluimos una lista de la bibliografía selecta que existe sobre Simmel.

\section{LIBROS}

Das Wesen der Materie nach Kants Physischer Monadologie, tesis doctoral, Berlín, 1881, 34 pp.

Über soziale Differenzierung. Soziologische und psychologische Untersuchungen, Duncker \& Humblot, Leipzig, 1890, 147 pp.

Die Probleme der Geschichtsphilosophie. Eine erkenntnistheoretische Studie, Duncker \& Humblot, Leipzig, 1892, 109 pp.

Einleitung in die Moralwissenschaft. Eine Kritik der ethischen Grundbegriffe, Hertz, Berlín, 1892, 426 pp.

Philosophie des Geldes, Duncker \& Humblot, Leipzig, 1900, 554 pp.

Kant. 16 Vorlesungen, gehalten an der Berliner Universität, Duncker \& Humblot, Leipzig, 1904, $181 \mathrm{pp}$.

Philosophie der Mode, Pan-Verlag, Berlín, 1905, 41 pp.

Kant und Goethe, Bard, Marquardt, Berlín, 1906, 71 pp.

Die Religion, Literarische Anstalt Rütten \& Loening, Frankfurt am Main, 1906, 79 pp.

Schopenhauer und Nietzsche. Ein Vortragszyklus, Duncker \& Humblot, Leipzig, 1907, 263 pp.

Soziologie. Untersuchungen über die Formen der Vergesellschaftung, Duncker \& Humblot, Leipzig, 1908, 782 pp.

Hauptprobleme der Philosophie, Göschen, Leipzig, 1910, 175 pp.

Philosophische Kultur. Gesammelte Essais, W. Klinkhardt, Leipzig, 1911, 319 pp.

Goethe, Klinkhardt \& Biermann, Leipzig, 1913, 264 pp.

Deutschlands innere Wandlung. Rede, Trübner, Strassburg, 1914, 14 pp.

Das Problem der historischen Zeit, Reuther \& Reichard, Berlín, 1916, 31 pp.

Rembrandt. Ein kunstphilosophischer Versuch, Kurt Wolff, Leipzig, 1916, 205 pp.

Grundfragen der Soziologie (Individuum und Gesellschaft), Göschen, Berlín y Leipzig, 1917, 103 pp.

Der Krieg und die geistigen Entscheidungen. Reden und Aufsätze, Duncker \& Humblot, München y Leipzig, 1917, 72 pp.

Der Konflikt der modernen Kultur. Ein Vortrag, Duncker \& Humblot, München y Leipzig, 1918, $48 \mathrm{pp}$.

Lebensanschauung. Vier metaphysische Kapitel, Duncker \& Humblot, München y Leipzig, 1918, $245 \mathrm{pp}$.

Vom Wesen des historischen Verstehens, Mittler, Berlín, 1918, 31 pp.

\section{ARTÍCULOS}

«Psychologische und ethnologische Studien über Musik», Zeitschrift für Völkerpsychologie und Sprachwissenschaft, 13, 1882, pp. 261-305.

«Dantes Psychologie», Zeitschrift für Völkerpsychologie und Sprachwissenschaft, 15, 1884, pp. 1869, 239-276.

«Über die Grundfrage des Pessimismus in methodischer Hinsicht», Zeitschrift für Philosophie und philosophische Kritik, núm. 90, 1887, pp. 237-247.

«Einige Bemerkungen über Goethes Verhältnis zur Ethik», Zeitschrift für Philosophie und philosophische Kritik, núm. 92, 1888, pp. 101-106. 
«Bemerkungen zu sozialethischen Problemen», Vierteljahrsschrift für wissenschaftliche Philosophie, 12, 1888, pp. 32-49.

«Michelangelo als Dichter», Vossische Zeitung, 8 septiembre 1889, Sonntagsbeilage, 36, pp. 6-9. "Zur Psychologie des Geldes», Jahrbuch für Gesetzgebung, Verwaltung und Volkswirtschaft im Deutschen Reich, 13, 1889, pp. 1251-1264.

«Moltke als Stilist», Berliner Tageblatt, 26 octubre 1890.

"Zur Psychologie der Frauen», Zeitschrift für Völkerpsychologie und Sprachwissenschaft, 20, 1890, pp. 6-46.

«Rembrandt als Erzieher», Vossische Zeitung, 1 junio 1890, Sonntagsbeilage, 22, pp. 7-10.

"Rudolf Euckens "Lebensanschauungen"», Vossische Zeitung, 14, 21, 28 junio 1891, Sonntagsbeilage, 24, pp. 1-3; 25, pp. 3-6; 26, pp. 4-8.

«Psychologische Glossen zur Strafgesetznovelle», Sozialpolitisches Zentralblatt, 1, 1892, p. 173.

«Ein jubiläum der Frauenbewegung», National-Zeitung, 27 noviembre 1892, Sonntagsbeilage, 48.

"Die Probleme der Geschichtsphilosophie (1892)», Vierteljahrsschrift für wissenschaftliche Philosophie, 16, 1892, p. 371.

«In Sachen der Moralwissenschaft», Vossische Zeitung, 27 marzo 1892, Sonntagsbeilage, 13, p. 11.

«Ein Wort über soziale Freiheit», Sozialpolitisches Zentralblatt, 1, 1892, pp. 333-335.

"Weltpolitik», Die Neue Zeit, 12, 1893/94 II, pp. 165-170.

«Die Bauernbefreiung in Böhmen", Allgemeine Zeitung, Munich, 14, 15 agosto 1894, Beilage.

"Der Militarismus und die Stellung der Frauen», Vossische Zeitung, 21, 28 octubre 1894, Sonntagsbeilage, 42, pp. 3-5; 43, pp. 2-4.

«Parerga zur Sozialphilosophie», Jahrbuch für Gesetzgebung, Verwaltung und Volkswirtschaft im Deutschen Reich, 18, 1894, pp. 257-265.

«Das Problem der Soziologie», Jahrbuch für Gesetzgebung, Verwaltung und, Volkswirtschaft im Deutschen Reich, 18, 1894, pp. 1301-1307.

«Die Verwandtenche», Vossische Zeitung, 3, 10 junio 1894, Sonntagsbeilage, 22, pp. 6-11; 23, pp. 8-12.

«Alpenreisen», Die Zeit, Viena, 4, 13 julio 1895.

«Über eine Beziehung der Selektionstheorie zur Erkenntnistheorie», Archiv für systematische Philosophie, 1, 1895, pp. 34-45.

"Gomperz "Griechische Denker" , Vossische Zeitung, 3 noviembre 1895, Sonntagsbeilage, 44, p. 11 y ss.

«Böcklins Landschaften», Die Zukunft, 12, 1895, pp. 272-277.

«Massenpsychologie», Die Zeit, Viena, 5, 23 noviembre 1895.

«Elisabeth Försters Nietzsche-Biographie», Berliner Tageblatt, 26 agosto 1895, Beilage Der Zeitgeist, 34.

«Zur Psychologie der Mode. Soziologische Studie», Die Zeit, Viena, 5, 12 octubre 1895.

"Zur Soziologie der Familie», Vossische Zeitung, 30 junio, 7 julio 1895, Sonntagsbeilage, 26, pp. 8-10; 27 pp. 8-10.

«Soziologische Ästhetik», Die Zukunft, 17, 1896, pp. 204-216.

«Der Frauenkongress und die Sozialdemokratie», Die Zukunft, 17, 1896, pp. 80-84.

«Das Geld in der modernen Kultur», Neue Freie Presse, Viena, 1896.

"Das Geld in der modernen Kultur», Zeitschrift des Oberschlesischen Berg- und Hüttenmännischen Vereins, 35, 1896, pp. 319-324.

«Berliner Gewerbeausstellung», Die Zeit, Viena, 8, 25 julio 1896.

«Berliner Kunstbrief», Die Zeit, Viena, 6, 21 marzo 1896.

"Zur Methodik der Sozialwissenschaft», Jahrbuch für Gesetzgebung, Verwaltung und Volkswirtschaft im Deutschen Reich, 20, 1896, pp. 575-585.

«Friedrich Nietzsche. Eine moralphilosophische Silhouette», Zeitschrift für Philosophie und philosophische Kritik, núm. 107, 1896, pp. 202-215.

«Über Plagiate. Offener Brief an Herrn Hermann Bahr», Die Zeit, Viena, 7, 27 junio 1896. 
«Skizze einer Willenstheorie», Zeitschrift für Psychologie und Physiologie der Sinnesorgane, 9, 1896, pp. 206-220.

«Was ist uns Kant?», Vossische Zeitung, 2, 9, 16 agosto 1896, Sonntagsbeilage, 31, pp. 1-4; 32, pp. 1-4; 33, pp. 7-10.

«Die Bedeutung des Geldes für das Tempo des Lebens», Neue Deutsche Rundschau, 8, 1897, pp. 111-122.

«Über Massenverbrechen», Die Zeit, Viena, 13, 2 octubre 1897.

«Soziale Medizin», Die Zeit, Viena, 10, 13 febrero 1897.

"Noch einmal "Der Pulsschlag”", Die Zeit, Viena, 11, 8 mayo 1897.

«Über den Unterschied der Wahrnehmungs- und der Erfahrungsurteile. Ein Deutungsversuch», Kantstudien, 1, 1897, pp. 416-425.

"Fragment aus einer "Philosophie des Geldes"», Zeitschrift für immanente Philosophie, 3, 1898, pp. 395-428, s.A 6.

"Stefan George. Eine kunstphilosophische Betrachtung», Die Zukunft, tomo 22, 1898, pp. 386396.

«Polymeter», Die Zeit, Viena, 16, 23 julio 1898.

«Recht. Ein Tragödien-Fragment. Von Simmel», Jugend, Munich, 5, 1898, II, p. 648 y ss.

"Die Rolle des Geldes in den Beziehungen der Geschlechter. Fragment aus einer "Philosophie des Geldes"”, Die Zeit, Viena, 14, 15, 22, 29 enero 1898; s. A 6.

«Rom. Eine ästhetische Analyse», Die Zeit, Viena, 15, 28 mayo 1898.

"Die Selbsterhaltung der sozialen Gruppe. Soziologische Studie», Jahrbuch für Gesetzgebung, Verwaltung und Volkswirtschaft im Deutschen Reich, 22, 1898, pp. 589-640.

"Fragment aus einer "Philosophie des Geldes"», Jahrbuch für Gesetzgebung, Verwaltung und Volkswirtschaft im Deutschen Reich, 23, 1899, pp. 813-854.

"Kant und Goethe», Allgemeine Zeitung, Munich, 3, 5, 6 junio 1899, Beilage, pp. 125-127.

"Zur Philosophie der Arbeit», Neue Deutsche Rundschau, 10, 1899, pp. 449-463.

«Von erfüllten Wünschen. Zwei sehr ähnliche Märchen von Simmel», Jugend, Munich, 4, 1899, II, p. 630.

"Einige Bemerkungen zu Schmollers "Grundriss der allgemeinen Volkswirtschaftslehre" , Allgemeine Zeitung, Munich, 28 octubre 1900, Beilage, p. 222.

"Metaphysik der Faulheit. Ein Satyrspiel zur Tragödie der Philosophie. Von G. Simmel», Jugend, Munich, 5, 1900, I, pp. 337-339.

«Sozialismus und Pessimismus», Die Zeit, Viena, 22, 3 febrero 1900.

"Zu einer Theorie des Pessimismus», Die Zeit, Viena, 22, 20 enero 1900.

"Ästhetik der Schwere», Berliner Tageblatt, 10 junio 1901, Der Zeitgeist, 23.

"Die ästhetische Bedeutung des Gesichts», Der Lotse. Hamburgische Wochenschrift für deutsche Kultur, vol. 1, Tomo 2, 1901, pp. 280-284.

"Stefan George. Eine kunstphilosophische Studie», Neue Deutsche Rundschau, 12, 1901, pp. 207-215.

«Zur Psychologie der Scham», Die Zeit, Viena, 29, 9 noviembre, 1901.

"Die beiden Formen des Individualismus», Das freie Wort. Frankfurter Halbmonatsschrift für Fortschritt auf allen Gebieten des geistigen Lebens, 1, 1901/1902, pp. 397-403.

«Philosophie des Geldes», Das freie Wort, 1, 1901/1902, pp. 170-174.

"Beiträge zur Erkenntnistheorie der Religion», Zeitschrift für Philosophie und philosophische Kritik, 119, 1902, pp. 11-22.

«Der Bildrahmen. Ein ästhetischer Versuch», Der Tag, Berlín, 1902, núm. 541.

"Weibliche Kultur», Neue Deutsche Rundschau, 13, 1902, pp. 504-515.

"Rodins Plastik und die Geistesrichtung der Gegenwart», Berliner Tageblatt, 29 septiembre 1902.

«Zum Fall Schmoller», Vossische Zeitung, 26 mayo 1902.

"Vom Heil der Seele», Das freie Wort, 2, 1902/1903, pp. 533-538.

"Vom Pantheismus», Das freie Wort, 2, 1902/1903, pp. 306-312.

«Zum Verständnis Nietzsches», Das freie Wort, 2, 1902/1903, pp. 6-11. 
«Die Grossstädte und das Geistesleben», en Die Grossstadt. Vorträge und Aufsätze zur Städteausstellung = Jahrbuch der Gehe-Stiftung, 9, 1903, pp. 185-206.

«Kant und die moderne Ästhetik», Berliner Tageblatt, 12, 19, octubre 1903, Der Zeitgeist 41, 42.

«Über räumliche Projektionen sozialer Formen», Zeitschrift für Sozialwissenschaft, 6, 1903, pp. 287-302.

«Uber ästhetische Quantitäten. Sitzungsbericht über einen Vortrag, geh. am 20 Januar 1903 in der Psychologischen Gesellschaft zu Berlin. Autorreferat», Zeitschrift für pädagogische Psychologie, Pathologie und Hygiene 5, 1903, pp. 208-212.

«Die ästhetischen Quantitäten», Berliner Tageblatt, 30, marzo 1903, Der Zeitgeist, 13.

«Soziologie der Konkurrenz», Neue Deutsche Rundschau, 14, 1903, pp. 1009-1023.

"Soziologie des Raumes», Jahrbuch für Gesetzgebung, Verwaltung und Volkswirtschaft im Deutschen Reich, 27, 1903, pp. 27-71.

«Die Lehre Kants von Pflicht und Glück», Das freie Wort, 3, 1903/1904. pp. 548-553.

«Bruchstücke aus einer Psychologie der Frauen», Der Tag, Berlín, 9 julio 1904.

«Über Geschichte der Philosophie», Die Zeit, Viena, 31, 28 mayo 1904.

"Wettbewerb oder Auftrag? Beantwortung einer Rundfrage», Die Zeit, Viena, 39, 16 abril 1904.

«Über die Zurechnungsfähigkeit perverser Verbrecher», Der Tag, Berlín, 16 julio 1904.

«Die Gegensätze des Lebens und der Religion», Das freie Wort, 4, 1904/1905, pp. 305-312.

«Das Abendmahl Lionardo da Vincis», Der tag, Berlín, 1905.

"Ästhetik des Porträts», Neue Freie Presse, Viena, 22 abril 1905.

«Das Ende des Streits», Die Neue Rundschau, vol. 16, Tomo 1, 1905, pp. 746-753.

«Schopenhauers Ästhetik und die moderne Kunstauffassung», Frankfurter Zeitung, 28, 29 agosto, 1906.

«Über die dritte Dimension in der Kunst», Zeitschrift für Ästhetik und allgemeine Kunstwissenschaft, 1, 1906, pp. 65-69.

«Kant und Goethe», Die Zukunft, 57, 1906, pp. 315-319.

«Nietzsche und Kant», Frankfurter Zeitung, 6 enero 1906.

«Psychologie der Diskretion», Der Tag, Berlín, 2, 4 septiembre 1906.

"Zur Soziologie der Armut», Archiv für Sozialwissenschaft und Sozialpolitik, 22, 1906, pp. 1-30.

«Bemerkungen über Goethe», Morgen. Wochenschrift über deutsche Kultur, 1, 1907, pp. 393-395.

«Das Christentum und die Kunst», Morgen, 1, 1907, pp. 234-243.

«Dankbarkeit. Ein soziologischer Versuch», Morgen, 1, 1907, pp. 593-598.

"Zur Philosophie der Herrschaft. Bruchstück aus einer Soziologie», Jahrbuch für Gesetzgebung, Verwaltung und Volkswirtschaft im Deutschen Reich, 31, 1907, pp. 439-471.

"Zur Soziologie des Adels. Fragment aus einer Formenlehre der Gesellschaft», Frankfurter Zeitung, 27 diciembre 1907.

«Soziologie der Sinne», Die Neue Rundschau, 18, 1907, II, pp. 1025-1036.

"Soziologie der Über- und Unterordnung», Archiv für Sozialwissenschaft und Sozialpolitik, 24 (núm. 6), 1907, pp. 477-546.

«Der Brief. Aus einer Soziologie des Geheimnisses», Österreichische Rundschau, 15, 1908, pp. 334-336.

«Der Mensch als Feind. Zwei Fragmente aus einer Soziologie» Morgen, 2, 1908, pp. 55-60; s. A 12.

«Zur Philosophie des Schauspielers», Morgen, 2, 1908, pp. 1685-1689.

«Das Problem des Stiles», Dekorative Kunst, 16, 1908, pp. 307-316.

"Psychologie des Schmuckes», Morgen, 2, 1908, pp. 454-459.

"Vom Realismus in der Kunst», Morgen, 2, 1908, pp. 992-998.

«Über Goethes und Kants moralische Weltanschauung. Aus einen Vorlesungszyklus», Der Tag, Berlín, 21 agosto 1908.

«Vom Wesen der Kultur», Österreichische Rundschau, 15, 1908, pp. 36-42.

"Über das Wesen der Sozial-Psychologie», Archiv für Sozialwissenschaft und Sozialpolitik, 26, 1908, pp. 285-291. 
"Beiträge zur Philosophie der Geschichte», Scientia. Rivista di scienza, 6 (Anno 3 II), 1909 II, pp. 345-351.

«Brücke und Tür», Der Tag, Berlín, 15 septiembre 1909.

"Fragmente eines Goethe-Buches... Aus dem Kapitel über Goethe und Kant», Österreichische Rundschau, 18, 1909, pp. 40-48; s. A 16.

"Beitrag Simmels zu "Religiose" Grundgedanken und moderne Wissenschaft. Eine Umfrage», Nord und Süd, Tomo 128, vol. 33, 1909 I, pp. 366-369.

"Die Kunst Rodins und das Bewegungsmotiv in der Plastik», Nord und Süd, Tomo 129, vol. 33, 1909 II, pp. 189-196.

«Psychologie der Koketterie», Der Tag, Berlín, 11, 12 mayo 1909.

«Der Siebente Ring», Münchner Neueste Nachrichten, 11 julio 1909.

«Die Zukunft unserer Kultur. Stimmen über Kulturtendenzen und Kulturpolitik», Frankfurter Zeitung, 14 abril 1909.

«Philosophie des Abenteuers», Der Tag, Berlín, 7, 8 junio 1910.

«Soziologie der Geselligkeit. Vortrag, geh. am Begrüssungsabend des I. Deutschen Soziologentages in Frankfurt a.M, 19. Okt. 1910», Frankfurter Zeitung, 21, 22 octubre 1910.

«Soziologie der Mahlzeit», Berliner Tageblatt, 10 octubre 1910, Der Zeitgeist 41.

"Vom Wesen der Philosophie», Frankfurter Zeitung, 6 febrero 1910.

"Zur Metaphysik des Todes», Logos. Internationale Zeitschrift für Philosophie der Kultur, 1, 1910/1911, pp. 57-70.

«Michelangelo. Ein Kapitel zur Metaphysik der Kultur», Logos, 1, 1910/1911, pp. 207-227.

"Diskussionsrede zum Vortrag von Ernst Troeltsch "Das stoisch-christliche Naturrecht und das moderne profane Naturrecht" 1911», Verhandlungen des I.Deutschen Soziologentages, 1910, Tübingen, 1911, pp. 204-206.

"Weibliche Kultur», Archiv für Sozialwissenschaft und Sozialpolitik, 33, 1911, pp. 1-36.

«Nietzsches Moral», Der Tag, Berlín, 4 mayo 1911.

"Die Persönlichkeit Gottes. Ein philosophischer Versuch», Zeitschrift für Theologie und Kirche, 21, 1911, pp. 251-269.

«Das Problem der religiösen Lage», en Weltanschauung. Philosophie und Religion in Darstellungen von Wilhelm Dilthey, Berlín: Reichl, 1911, pp. 327-340.

"Soziologie der Geselligkeit», Schriften der Deutschen Gesellschaft für Soziologie. Serie I, Tomo 1, Verhandlungen des I. Deutschen Soziologentages, (1910) 1911, pp. 1-16.

«Der Begrift und die Tragödie der Kultur», Logos, 2, 1911/1912, pp. 1-25.

"Das Relative und das Absolute im Geschlechterproblem», Frauen-Zukunft, 2, 1911/1912, I, pp. 157-172, 253-265.

"Goethe und die Frauen», St. Petersburger Monatsblatt, 1912, núm. 463.

"Goethe und die Frauen», Ostsee-Zeitung, Stettin, 28 septiembre 1912.

"Goethe und die Frauen», Potsdamer Tageszeitung, 5 octubre 1912.

"Goethes Individualismus», Logos, 3, 1912, pp. 251-274.

"Goethes Liebe», Frankfurter Zeitung, 21, julio 1912.

«Polarität und Gleichgewicht bei Goethe», Vossische Zeitung, 19 septiembre 1912.

"Über einige gegenwärtige Probleme der Philosophie», Vossische Zeitung, 17 noviembre 1912.

«Goethes Rechenschaft», Der Tag, Berlín, 4 febrero 1912.

«Der Schauspieler und die Wirklichkeit», Berliner Tageblatt, 7 enero 1912.

«Die Stetigkeit in Goethes Weltbild», Der Tag, Berlín, 9 octubre 1912.

«Über Takt. Soziologie der Geselligkeit», Frankfurter Zeitung, 22 octubre 1912.

«Das Verhältnis von Leben und Schaffen bei Goethe», Der Tag, Berlín, 22, 23 marzo 1912.

"Die Wahrheit und das Individuum. Aus einem Goethebuch», Logos, 3, 1912, pp. 15-28; s. A 16.

"Philosophie der Landschaft», Die Güldenkammer. Norddeutsche Monatshefte, 3, 1912/ 1913, pp. 635-644.

"Das individuelle Gesetz. Ein Versuch über das Prinzip der Ethik», Logos, 4, 1913, pp. 117-160. «An Herrn Professor Karl Lamprecht», Die Zukunft, 83, 1913, pp. 230-234. 
«Die Hamburgische Universität», Der Tag, Berlín, 12 febrero 1913.

«Henri Bergson», Die Güldenkammer, 4, 1913/1914, pp. 511-525.

"Goethes Gerechtigkeit", Der Greif. Cotta'sche Monatsschrift, 1, 1913/1914 II, pp. 381-387.

"Das Problem des Schicksals», Die Geisteswissenschaften, 1, 1913/1914, pp. 112-115.

"L'Art pour l'Art», Der Tag, Berlín, 4 enero 1914.

"Goethe und die Jugend», Der Tag, Berlín, 6 agosto 1914.

«Der Fall Jastrow», Die Zukunft, tomo 89, vol. 23 IV, 1914, pp. 33-36.

«Rembrandts religiöse Kunst», Frankfurter Zeitung, 30 junio, 1 julio 1914.

«Rembrandt und die Schönheit», Vossische Zeitung, 25 diciembre 1914.

"Aus einer Aphorismensammlung", Der Kunstfreund. Zeitschrift der Vereinigung der Kunstfreunde, 2, 1914/1915, pp. 284-286.

"Bergson und der deutsche "Zynismus" , Internationale Monatsschrift für Wissenschaft, Kunst und Technik, 9, 1914/1915, pp. 197-200.

«Rembrandtstudie», Logos, 5, 1914/1915, pp. 1-32.

«Einheit und Zwiespalt. Zeitgemässes in Goethes Weltanschauung», Berliner Tageblatt, 7 noviembre 1915.

«Europa und Amerika. Eine weltgeschichtliche Betrachtung», Berliner Tageblatt, 4 julio 1915.

«Die Idee Europa», Berliner Tageblatt, 7 marzo 1915.

«Geld und Nahrung», Der Tag, Berlín, 28 marzo 1915.

"Vom Tode in der Kunst. Nach einem Vortrag», Frankfurter Zeitung, 2 abril 1915.

"Bruchstücke aus einer Philosophie der Kunst», Göteborg: Wettergren \& Kerber, 1916, pp. 15-26

«Die Dialektik des deutschen Geistes», Der Tag, Berlín, 28 septiembre 1916.

"Gestalter und Schöpfer», Der Tag, Berlín, 10 febrero 1916.

«Die Krisis der Kultur», Frankfurter Zeitung, 13 febrero 1916.

"Goethes Liebe. Referat über den gleichnamigen Vortrag, den Simmel im Auditorium maximun der Berliner Universität gehalten, nach dem Bericht des "Berliner Tageblatts" vom 17 marzo 1916", Die neue Generation, 12, 1916, pp. 101-103.

"Wandel der Kulturformen», Berliner Tageblatt, 27 agosto 1916.

«Der Fragmentcharakter des Lebens. Aus den Vorstudien zu einer Metaphysik», Logos, 6, 1916/1917, pp. 29-40.

«Vorformen der Idee. Aus den Studien zu einer Metaphysik», Logos, 6, 1916/1917, pp. 103-141. «Erinnerung an Rodin», Vossische Zeitung, 27 noviembre 1917.

«Eine Fastenpredigt. Von dem Opfer der Wohlhabenden», Frankfurter Zeitung, 18 marzo 1917.

"Das Goethebuch», Die Neue Rundschau, 28, 1917, pp. 254-264.

«Über die Karikatur», Der Tag, Berlín, 27 febrero 1917.

«Die historische Formung», Logos, 7, 1917/1918, pp. 113-152.

"Individualismus», Marsyas, 1, 1917/1919, pp. 33-39.

«Das problem des Porträts», Die Neue Rundschau, 29, 1918, pp. 1336-1344.

"Germanischer und Klassisch-romanischer Stil», Der Tag, Berlín, 2 marzo 1918.

«Aus Georg Simmels nachgelassenem Tagebuch», Logos, 8, 1919/1920, pp. 121-151.

"Ausbildung der Individualität im Zusammenhang mit der Erweiterung der Gruppe», Vom sozialen Leben und Werden unserer Zeit. Zwölf Aufsätze zeitgenössischer Schriftsteller. Compilado por Elisabeth Herbig, Bielefeld u. Leipzig: Velhagen \& Klasing, 1920.

"Gedanken aus Georg Simmels Nachlass", Geisteskultur und Volksbildung, 29, 1920, p. 294.

"Zur Philosophie des Schauspielers. Aus dem Nachlass herausgegeben», Logos, 9, 1920/1921, pp. 339-362.

«Fragment über die Liebe. Aus dem Nachlass Georg Simmels», Logos, 10, 1921/1922, pp. 1-54.

«Exkurs über den platonischen und den modernen Eros», Logos, 10, 1921/1922, pp. 25-28.

«Über Freiheit. Bruchstücke aus dem Nachlass von Georg Simmel», Logos, 11, 1922/1923, pp. 1-30.

"Gesamteinleitung zu Goethes Sämtlichen Werken», Compilado por Curt Noch y Paul Wiegler (Pandora-Klassiker), tomo 1, Berlín: Ullstein, 1923, pp. 11-97.

"Aus dem nachgelassenen Tagebuch», Geistige Werte der Deutschen. Neue, gänzlich umgestaltete u. erw. Ausgabe, hrsg. von Werner Schingnitz, Darmstadt: Reichl, 1926, pp. 542-555. 
"Aus Georg Simmels nachgelassener Mappe "Metaphysik", Gertrud Simmel», Àus unbekannten Schriften. Festgabe für Martin Buber zum, 50, Geburtstag', 1928, pp. 221-226.

"Die Alpen», «Deutsche Geisteswelt», Compilado por Arthur Hübscher, asesoramiento de Theodor Kiener, tomo 2, Von Schopenhauer bis Heisenberg", Darmstadt-Baden Baden-Genf: Holle Verlag, 1953, pp. 165-174.

"Die Transzendenz des Lebens», Deutscher Geist. Ein Lesebuch aus zwei jahrhunderte, Suhrkamp, Berlín y Frankfurt a. M., 1953, tomo 2, pp. 515-539.

«Das Individuum und die Freiheit», Brücke und Tür, 1957, pp. 260-269.

«Briefwechsel Simmel-Rilke», St. Galler Tageblatt, 28 febrero 1958.

«Brücke und Tür», Christ und Welt, Berliner Ausgabe, Stuttgart. 27 marzo 1958.

"Zwiegespräche eines Philosophen mit einem Dichter. Zum 100. Geburtstage Georg Simmels. Aus seinem bisher unveröffentlichten Briefwechsel mit Rainer Maria Rilke», Der Tagesspiegel, Berlín, Feuilleton, 2 marzo 1958.

\section{COMPILACIONES}

Zur Philosophie der Kunst. Philosophische und kunstphilosophische Aufsätze, G. Simmel (ed.), Kiepenheuer, Postdam, 1922, $173 \mathrm{pp}$.

Schulpädagogik. Vorlesungen, K. Hauter (ed.), Zickfeldt, Osterwieck/Harz, 1922, 134 pp.

Fragmente und Aufsätze aus dem Nachlass und Veröffentlichungen der letzten Jahre, G. Kantorowicz (ed.), Drei Masken, München, 1923, 304 pp.

Schopenhauer und Nietzsche: ein Vortragszyklus, Duncker und Humblot, München, 1923, 192 pp.

Rembrandtstudien, Schwabe, Basel, 1953, 72 pp.

Rembrandtstudien. Sonderausgabe, Wissenschaftliche Buchgemeinschaft, Darmstadt, 1953, $72 \mathrm{pp}$.

Brücke und Tür. Essays des Philosophen zur Geschichte, Religion, Kunst und Gesellschaft, M. Landmann (ed.), K. F. Koehler, Stuttgart, 1957, 281 pp.

Buch des Dankes an Georg Simmel, Briefe, Erinnerungen, Bibliographie, K. Gassen, M. Landmann (ed.), Duncker \& Humblot, Berlín, 1958.

Wolff, K. H. (ed.), Georg Simmel. 1858-1918. A collection of essays, with translations, and a bibliography, Ohio State University, 1959.

Wolff, K. H., The sociology of Georg Simmel, University of Chicago Press, Chicago, 1964.

Coser, L. A. (ed.), Georg Simmel, Englewood Cliffs, N. J., Prentice Hall, 1965.

Schriften zur Philosophie und Soziologie der Geschlechter, H.-J. Dahme, K. C. Köhnke (ed.), Suhrkamp, Frankfurt am Main, 1985.

Philosophisches Kultur: über das Abenteuer, die Geschlechter und die Krise der Moderne. Gesammelte Essais, Wagenbach, Berlín, 1986, 252 pp.

Schriften zur Soziologie: eine Auswahl, H.-J. Dahme, O. Rammstedt (ed.), Suhrkamp, Frankfurt am Main, 1986, 308 pp.

Das individuelle Gesetz: philosophische Exkurse, M. Landmann (ed.), Suhrkamp, Frankfurt am Main, 1987, 275 pp.

Gesammelte Schriften zur Religionssoziologie, H. J. Helle (ed.), Duncker \& Humblot, Berlín, 1989, 180 pp.

Vom Wesen der Moderne. Essais zur Philosophie und Asthetik, W. Jung (ed.), Jurius, Hamburg, 1990, $360 \mathrm{pp}$.

Frisby, D. (ed.), Georg Simmel. Critical Assessments, 3 volúmenes, Routledge and Keegan Paul, Londres, 1994. 


\section{OBRAS COMPLETAS (Gesamtausgabe)}

\section{Tomo I}

Das Wesen der Materie (1881). Abhandlungen 1882-1884. Rezensionen 1883-1901, K. C. Köhnke (ed.), Suhrkamp, Frankfurt am Main.

\section{Tomo II}

Aufsätze 1887-1980. Über sociale Differenzierung (1890). Die Probleme der Geschichtsphilosophie (1892), H.-J. Dahme (ed.), Suhrkamp, Frankfurt am Main, 1989.

Tomo III

Einleitung in die Moralwissenschaft. Eine Kritik der ethischen Grundbegriffe (primera parte), K. C. Köhnke (ed.), Suhrkamp, Frankfurt am Main, 1989.

\section{Tomo IV}

Einleitung in die Moralxissenschaft. Eine Kritik der ethischen Grundbegriffe (segunda parte), K. C. Köhnke (ed.), Suhrkamp, Frankfurt am Main, 1991.

Tomo $V$

Aufsätze und Abhandlungen 1894-1900, H.-J. Dahme, D. P. Frisby (ed.), Suhrkamp, Frankfurt am Main, 1992.

Tomo VI

Philosophie des Geldes (1900/1907), D. P. Frisby, K. C. Köhnke (ed.), Suhrkamp, Frankfurt am Main, 1989.

Tomo VII

Aufsätze und Abhandlungen 1901-1908 (primera parte), R. Kramme, A. Rammstedt, O. Rammstedt (ed.), Suhrkamp, Frankfurt am Main, 1995.

Tomo VIII

Aufsätze und Abhandlungen 1901-1908 (segunda parte), A. Cavalli, V. Krech (ed.), Suhrkamp, Frankfurt am Main, 1993.

Tomo IX

Kant (1904/1913/1918). Die Probleme der Geschichtsphilosophie, 2. Fassung (1905/1907), G. Oakes, K. Röttgers (ed.), Suhrkamp, Frankfurt am Main, 1997.

\section{Tomo $X$}

Philosophie der Mode (1905). Die Religion (1906/1912). Kant und Goethe (1906/1916). Schopenhauer und Nietzsche (1907), M. Behr, V. Krech, G. Schmidt (ed.), Suhrkamp, Frankfurt am Main, 1995.

Tomo XI

Soziologie. Untersuchungen über die Formen der Vergesellschaftung (1908), O. Rammstedt (ed.), Suhrkamp, Frankfurt am Main, 1992.

\section{Tomo XII}

Aufsätze und Abhandlungen 1909-1918 (primera parte), Suhrkamp, Frankfurt am Main.

Tomo XIII

Aufsätze und Abhandlungen 1909-1918 (segunda parte), Suhrkamp, Frankfurt am Main.

Tomo XIV

Hauptprobleme der Philosophie (1910/1927). Philosophische Kultur (1911/1918), R. Kramme, O. Rammstedt (ed.), Suhrkamp, Frankfurt am Main, 1996.

\section{Tomo $X V$}

Goethe (1913). Deutschlands innere Wandlung (1914). Das Problem der historischen Zeit (1916). Rembrandt (1916), Suhrkamp, Frankfurt am Main.

Tomo XVI

Grundfragen der Soziologie (1917). Der Krieg und die geistigen Entscheidungen (1917). Der Konflikt der modernen Kultur (1918). Vom Wesen des historischen Verstehens (1918). Lebensanschauung (1918), Suhrkamp, Frankfurt am Main.

\section{Tomo XVII}

Miszellen. Glossen, Stellungnahmen, Umfrageantworten, Lesebriefe, Diskussionsbeiträge 18891917. Anonyme und pseudonyme Veröffentlichungen 1888-1917. Beiträge aus der Jugend 1897-1916, Suhrkamp, Frankfurt am Main. 


\section{Tomo XVIII}

Englischsprachige Veröffentlichungen 1893-1910, Suhrkamp, Frankfurt am Main.

\section{Tomo XIX}

Französisch- und italienischsprachige Veröffentlichungen 1894-1903. Mélanges de philosophie relativiste: contribution à la culture philosophique (1912), Suhrkamp, Frankfurt am Main.

Tomo XX

Drucke aus dem NachlaB. Nicht ermittelte Erstdrucke. Ungedrucktes, Suhrkamp, Frankfurt am Main.

Tomo XXI

Nachschriften und Referate von Vorlesungen und Vorträgen, Suhrkamp, Frankfurt am Main.

Tomo XXII

Briefe (primera parte), Suhrkamp, Frankfurt am Main.

Tomo XXIII

Briefe (segunda parte), Suhrkamp, Frankfurt am Main.

Tomo XXIV

Gesamtbibliographie und register, Suhrkamp, Frankfurt am Main.

\section{TRADUCCIONES}

\section{Lengua castellana}

\section{A) España}

Schopenhauer y Nietzsche, traducción de José R. Pérez-Bances, Ed. Beltrán, Madrid, 1915, 267 pp.

«Filosofía de la moda», Revista de Occidente, Año 1, Tomo 1, 1923, pp. 42-66, 211-230.

"Lo masculino y lo femenino. Para una psicología de los sexos», Revista de Occidente, Año 1, Tomo 2, 1923, pp. 218-236, 336-363.

«El asa (Ensayos estéticos)», Revista de Occidente, Año 2, Tomo 4, 1924, Nr. 12.

"Cultura femenina y otros ensayos», traductores Eugenio Imaz, José R. Pérez Bances, Manuel García Morente y Fernando Vela, Biblioteca Revista de Occidente, Madrid, 1934, 329 pp. (Incluye: Introducción - Cultura femenina - Filosofía de la coquetería - Lo masculino y lo femenino - La aventura - Filosofía de la moda - Concepto y tragedia de la cultura - Las ruinas - El problema de la situación religiosa - La personalidad de Dios - Los Alpes - Miguel Ángel - Rodin - El asa.)

"Cultura femenina. Filosofía de la coquetería», trad. por Ambrosio Fernández, Religión y Cultura, Madrid, Año7 II, Tomo 26, 1924, p. 441.

"Cultura Femenina», Revista de Occidente, Año 3, Tomo 7, 1925, pp. 273-301; Tomo 8, 1925, pp. 170-199.

"Filosofía de la coquetería. Filosofía de la moda. Lo masculino y lo femenino y otros ensayos», Biblioteca de la Revista de Occidente, Madrid, 1924, p. 229; 5. a ed., 1945.

«Las ruinas», Revista de Occidente, Año 2, Tomo 4, 1924, Nr. 12.

«El problema de la situación religiosa», Revista de Occidente, Año 3, Tomo 10, 1925, pp. 290-308.

«Fidelidad y gratuidad», Revista de Occidente, Año 4, Tomo 14, 1926, pp. 292-317.

"Problemas fundamentales de la filosofía», traducción de Fernando Vela, Biblioteca de la Revista de Occidente, Madrid, 1946, 206 pp.

La filosofía del dinero, traducción de Ramón García Cotarelo, Instituto de Estudios Políticos, Madrid, 1977, 663 pp.

«Sociología I: estudios sobre las formas de socialización», traducción de José R. Pérez Bances, Revista de Occidente, Madrid, 1977. (Incluye: El problema de la Sociología - La cantidad en los grupos sociales - La subordinación - La Lucha - El secreto y la sociedad secreta.) 
«Sociología II: estudios sobre las formas de socialización», traducción de José R. Pérez Bances, Revista de Occidente, Madrid, 1977. (Incluye: El cruce de los círculos sociales - El pobre La autocondenación de los grupos sociales - El espacio y la sociedad - La ampliación de los grupos y la formación de la individualidad.)

Sociologia I: estudios sobre las formas de socialización, Alianza, Madrid, 1986, $424 \mathrm{pp}$.

Sociología II: estudios sobre las formas de socialización, Alianza, Madrid, 1986, $383 \mathrm{pp}$

El individuo y la libertad: ensayos de crítica de la cultura, traducción de Salvador Mas Torres, Península, Barcelona, 1986, 284 pp. (Incluye una traducción completa de la versión de Brücke und Tür compilada por Landmann y Susman, excepto los dos ensayos «Goethe y la juventud» e "Individualismo», y se recoge el trabajo "Estética sociológica», publicado originalmente en 1896.)

Sobre la aventura: ensayos filosóficos, traducido por Gustavo Muñoz y Salvador Mas Torres, Península, Barcelona, 1988, 285 pp. (Incluye una traducción completa del original alemán de Philosophische Kultur, 1911.)

Rembrandt, Artes Gráficas Soler, Valencia, 1996, 268 pp.

«El problema del estilo», trad. J. Almaraz, en REIS, 84, 1998, pp. 319-326.

Cultura femenina y otros ensayos, traductora Genoveva Dieterich, Alba, Barcelona, 1999. (Incluye: Introducción - La aventura - La moda - Lo relativo y lo absoluto en el problema de los sexos - La coquetería - El concepto y la tragedia de la cultura - Cultura femenina.)

\section{B) Sudamérica}

El conflicto de la cultura moderna, traducido por Carlos Astrada, Universidad Nacional de Córdoba, Argentina, 1923.

«Filosofía de la aventura», Sagitario, 2, ed. de la Plata, Buenos Aires, 1925, pp. 41-56.

Cultura femenina y otros ensayos (Cultura femenina. Filosofía de la coquetería. Lo masculino y lo femenino. Filosofía de la moda), Espasa Calpe Argentina, Buenos Aires, 1938 (Col. Austral 48), 3. ${ }^{\mathrm{a}}$ ed., 1941; 1946 (5. ${ }^{\mathrm{a}}$ ed.).

Sociología, José R. Pérez Bances, Espasa Calpe Argentina, Buenos Aires, 1939.

Schopenhauer y Nietzsche, traducido por Francisco Ayala, Editorial Schapire, Buenos Aires, 1944.

Problemas fundamentales de la filosofía, traducido por S. Molinari y E. Suchulzen, Editorial de La Plata, Buenos Aires, 1947.

Goethe. Kant y Goethe. Para la historia de la concepción moderna del mundo, traducido por José Rovira Armengol, Editorial Nova, Buenos Aires, 1949, 312 pp.

Intuición de la vida. Cuatro capitulos de metafísica, traducido por José Rovira Armengol, Editorial Nova, 1950, 227 pp. (Incluye la traducción completa del volumen alemán Lebensanschauung. Vier metaphysische Kapitel.)

Problemas de filosofia de la historia. El tiempo histórico. La configuración histórica, traducido por Elsa Tabernig, Editorial Nova, Buenos Aires, 1950, 266 pp.

Rembrandt. Ensayo de filosofía del arte, Emilio Estiú, Editorial Nova, Buenos Aires, 1950, 220 pp.

Cultura femenina, Espasa Calpe Mexicana, México, 1961, 143 pp.

\section{Lengua inglesa}

Conflict. The Web of Group-affiliations, The Free Press, Glencoe, 1955, 195 pp.

Conflict. The Web of Group-affiliations, Free Press, New York, 1964, 195 pp.

On Individuality and Social Forms: Selected Writings, D. N. Levine (ed.), University of Chicago Press, Chicago, 1971, 395 pp.

The Philosophy of Money, Routledge, London, 1990 (2. a ed.). 
"Moral Deficiences as Determining Intellectual Functions», en International Journal of Ethics, 3, 1893, pp. 490-507.

"The Problem of Sociology», en Annals of the American Academy of political and Social Science, 6, 1895, pp. 412-423.

"Superiority and Subordination as Subject-matter for Sociology", en The American Journal of Sociology, 2, 1896/1897, pp. 167-189.

"The Persistence of Social Groups", en The American Journal of Sociology, 3, 1897/1898, pp. 662-698, 829-836; 4, 1898/1899, pp. 35-50.

"A Chapter in the Philosophy of Value. A Fragment from a Volume Entitled "The Philosophy of Money" ", en The American Journal of Sociology, 5, 1899/1900, pp. 577-603.

"Tendencies in German Life and Thought Since 1870», en International Monthly, 5, New York, 1902, pp. 93-111, 166-184.

"The Number of Members as Determining the Sociological form of the Group», en The American Journal of Sociology, 8, 1902/1903, pp. 1-46, 158-196.

«The Sociology of Conflict», en The American Journal of Sociology, 9, 1903/1904, pp. 490-525.

«Fashion», en International Quarterly, 10, New York, 1904, pp. 130-155.

"A Contribution to the Sociology of Religion", en The American Journal of Sociology, 11, 1905/1906, pp. 359-376.

"The Sociology of Secrecy and of the Secret Societies», en The American Journal of Sociology, 11, 1905/1906, pp. 441-498.

"The Problem of Sociology», en The American Journal of Sociology, 15, 1909/1910, pp. 289-320.

"How is Society Possible?», en The American Journal of Sociology, 16, 1910, pp. 372-391.

"The Sociological Significance of the "Stranger" , en Introduction to the Science of Sociology, 1921, pp. 322-327.

"Sociology of the Senses: Visual Interaction», en Introduction to the Science of Sociology, 1921, pp. 356-361.

"Money and Freedom», en Introduction to the Science of Sociology, 1921, 552-553.

"Social Interaction as the Definition of the Group in Time and Space», en The American Journal of Sociology, 3, 1897/1898, y 15, 1909/1910.

"The Metropolis and Mental Life», en Syllabus and Selected Readings, 5. a ed., University of Chicago, Sept. 1936, pp. 221-238.

"The Sociology of Sociability», en The American Journal of Sociology, 55, 1949/1950, pp. 254261.

"Contribution to the Sociology of Religion", en The American Journal of Sociology, 60, 1955, pp. $1-18$.

"Superiority and Subordination in Social Relationship», en Sociological Theory. Present Day Sociology from the past, Knopf, New York, 1956, pp. 180-204.

"Knowledge and Ignorance», en Sociological Theory. Present Day Sociology from the past, Knopf, New York, 1956, pp. 205-226.

"The Persistence of Social Groups», en Sociological Theory. Present Day Sociology from the past, Knopf, New York, 1956, pp. 364-398.

«The Sociology of Georg Simmel», Kurt. H. Wolff, The Free Press, LXIV, 1950, p. 445.

\section{Lengua francesa}

Mélanges de philosophie relativiste: contribution à la culture philosophique, Alcan, París, 1912, 268 pp.

Les problèmes de la philosophie de l'histoire: une étude d'épistémologie, PUF, París, 1984, 244 pp.

Philosophie de l'amour, Rivage, Paris, 1988, 195 pp.

"La différentiation sociale», en Revue Internationale de Sociologie, 2, 1894, pp. 198-213. 
"L'influence du nombre des unités sociales sur les caractères des sociétés», en Annales de l'Institut International de Sociologie, 1, 1894, pp. 373-385.

«Le problème de la sociologie», en Revue de Métaphysique et de Morale, 2, 1894, pp. 497-504.

"Sur quelques relations de la pensée théorique avec les intéréts pratiques», en Revue de Métaphysique et de Morale, 4, 1896, pp. 160-178.

«Comment les formes sociales se mantiennent», en L'Année Sociologique, 1, 1896/1897, pp. 71109.

\section{Lengua italiana}

«Il problema della sociologia», Riforma sociale, Fasc. 7, Anno 6, 1899, ser. 2.

I problemi fondamentali della filosofia, Vallecchi, Florencia, 1922, $230 \mathrm{pp}$.

Il Relativismo, Carabba, Lanciano, 1922, 138 pp.

Schopenhauer e Nietzsche, Paravia, Torino, 1923, 156 pp.

Il conflitto della civiltà moderna, Bocca, Torino, 1925, 75 pp.

L'etica e i problemi della cultura moderna, Guida, Nápoles, 1968, 90 pp.

Filosofía dell denaro, traducción de Alessandro Cavalli, Roma, 1984.

Sociologia, traducción de Alessadro Cavalli, Roma, 1989.

\section{ESCRITOS SOBRE SIMMEL}

ABeL, Th., Systematic Sociology in Germany, New York, 1929.

— "The Contribution of Georg Simmel. A reappraisal», American Sociological Review, 24, 1959, pp. 473-479.

AdLER, M., Georg Simmels Bedeutung für die Geistesgeschichte, Viena, 1919.

AdoRno, Th. W., «Henkel, Krug und frühe Erfahrung», en Ernst Bloch zu Ehren, Frankfurt/M., 1965, pp. 9-20.

Altaraz, I., Reine Soziologie. Darstellung und Kritik der typischen Versuche zur Schaffung einer philosophischen Sozialwissenschaft, Diss, Berlín, 1918.

Altmann, S. P., "Die Philosophie des Geldes», Deutschland. Monatsschrift für die gesamte Kultur, 3, 1904, pp. 88-104; 213-119.

Aron, R., Deutsche Soziologie der Gegenwart, Stuttgart, 1969.

Axelrod, Ch. D., "Toward an Appreciation of Simmel's Fragmentary Style», The Sociological Quarterly, 18, 1977, pp. 185-196.

Axelrod, Ch. D., Studies in Intellectual Breakthrough. Freud, Simmel, Buber, Amherst, 1979.

Bauer, I., Die Tragik in der Existenz des modernen Menschen bei Georg Simmel, Diss, München, 1961.

Becher, H.-J., Georg Simmel. Die Grundlagen seiner Soziologie, Stuttgart, 1971.

Becker, H., "On Simmel's "Philosophy of Money" ", en Wolff, K. H. (ed.), Georg Simmel. 1858-1918. A collection of essays, with translations, and a bibliography, Ohio State University, 1959, pp. 216-232.

Bentley, A. F., "Simmel, Durkheim, Ratzenhofer», American Journal of Sociology, 32, 1926/1927, pp. 250-256.

Bernhard, E., "Georg Simmel als Soziologe und Sozialphilosoph», Die Tat, 5, 1914, pp. 10801086.

Bevers, A. M., Dynamik der Formen bei Georg Simmel. Eine Studie über die methodische und theoretische Eingeit eines Gesamtwerkes, Berlín/Munich, 1985.

Blumenberg, H., "Geld oder Leben. Eine metaphorologische Studie zur Konsistenz der Philosophie Georg Simmels», en Böhringer, H., y Gründer, K. (eds.), Ästhetik und Soziologie um die jahrhundertwende: Georg Simmel, Frankfurt/M., 1976, pp. 121-134. 
BöHringer, H., "Spuren Von spekulativem Atomismus in Simmels formaler Soziologie», en Böhringer, H., y Gründer, K. (eds.), Ästhetik und Soziologie um die Jahrhundertwende: Georg Simmel, Frankfurt/M., 1976, pp. 105-117.

Böhringer, H., y GrÜnder, K. (eds.), Ästhetik und Soziologie um die jahrhundertwende: Georg Simmel, Frankfurt/M., 1976.

BoHner, H., Untersuchungen zur Entwicklung der Philosophie Georg Simmels, Diss, Freiburg i. $\mathrm{Br}, 1930$.

Bouglé, C., "Les sciences sociales en Allemagne: Georg Simmel», Revue de Métaphysique et de Morale, 2, 1894, pp. 329-355.

- "The Sociology of Georg Simmel», en Coser, L. A. (ed.), Georg Simmel, Englewood Cliffs, N. J., 1912, pp. 58-63.

Brinkmann, H., Methode und Geschichte. Die Analyse der Entfremdung in Georg Simmels "Philosophie des Geldes», Giessen., 1974.

Christian, P., Einheit und Zwiespalt. Zum hegelianisierenden Denken in der Philosophie und Soziologie Georg Simmels, Berlín, 1978.

Coser, L. A., Theorie sozialer Konflikte, Neuwied, 1956.

— «Georg Simmel's Style of Work», American Journal of Sociology, 63, 1958, pp. 635-640.

— "The Sociology of poverty», Social Problems, 13, 1965/1966, pp. 140-148.

- Masters of Sociological Thought. Ideas in Historical and Social Context, New York, 1971.

- "Georg Simmel's Neglected Contributions to the Sociology of Women», Signs. Journal of Women in Culture and Society, 2, 1977, pp. 869-876.

- (ed.) «Georg Simmel», Englewood Cliffs, N. J.

DaHme, H.-J., Soziologie als exakte Wissenschaft Georg Simmels Ansatz und seine Bedeutung in der gegenwärtigen Soziologie, tomo 2, Stuttgart, 1981.

Dahme, H.-J., y Rammstedt, O., Georg Simmel und die Moderne. Neue Interpretationen und Materialien, Frankfurt am Main, 1984.

Davis, M. S., "Georg Simmel and the Aesthetics of Social Reality", Social Forces, 51, 1972/1973, pp. 320-329.

Dieterich, O., Georg Simmel und seine Bedeutung für die Nationalökonomie, Diss, Freiburg i. Br., 1921.

Duncan, H. D., «Simmel's Image of Society», en Wolff, K. H. (ed.), Georg Simmel. 1858-1918. A collection of essays, with translations and a bibliography, Ohio State University, 1959, pp. 100-118.

DurKheim, E., «La sociologia ed il suo dominio scientifico», Revista Italiana di Sociologia, 4, 1900, pp. 127-148.

EISERMANN, G., «Georg Simmel», en A. Silbermann (ed.), Klassiker der Kunstsoziologie, Munich, 1979, pp. 64-84.

EKHART, L., Georg Simmels philosophische Begründung seiner Soziologie, Diss, Graz, 1956.

EulenburG, F., "Simmels Probleme der Geschichtsphilosophie (Neuere Geschichtsphilosophie III C 1)", Archiv für Sozialwissenschaft und Sozialpolitik, 29, 1909, pp. 169-197.

Featherstone, M., Georg Simmel, London, 1991.

Featherstone, M., y Frisby, D., Simmel on Culture, Sage, Londres, 1997.

Freund, J., "Der Dritte in Simmels Soziologie», en Böhringer, H., y Gründer, K. (eds.), Ästhetik und Soziologie um die jahrhundertwende: Georg Simmel, Frankfurt/M., 1976, pp. 90-104.

FreYer, H., Soziologie als Wirklichkeitswissenschaft, Leipzig-Berlín, 1930.

FrisbY, D., "Introduction to the Translation», en G. Simmel, The Philosophy of Money, Londres, 1978, pp. 1-49.

- Sociological Impressionism. A Reassessment of Georg Simmel's Social Theory, Londres, 1981.

- Fragmente der Moderne. Georg Simmel - Siegfried Kracauer - Walter Benjamin, Rheda-Wiedenbrück, 1989.

- Simmel and Since. Essays on Georg Simmel's Theory, Londres, 1992.

FrisCHEISEN-KÖHLER, M., "Georg Simmel», en Kant-Studien, 24, 1920, pp. 1-51.

Frost, W., "Die Soziologie Simmels», en Acta Universitatis Latviensis (Riga), 12 (1925), pp. 219-313; 13 (1926), pp. 149-225. 
Gassen, K., y Landmann, M. (ed.), Buch des Dankes an Georg Simmel. Briefe, Erinnerungen, Bibliographie, Berlín, 1958.

GephaRT, W., «Emile Durkheim: Die elementaren Formen des religiösen Lebens. Oder: Das "unheimliche Verhältnis" von Durkheim zu Simmel», en Soziologische Revue, 5, 1982, pp. 11-17.

- "Soziologie im Aufbruch. Zur Wechselwirkung von Durkheim, Schäffle, Tönnies und Simmel», en Kölner Zeitschrift für Soziologie und Sozialpsychologie, 34, 1982, pp. 1-25.

GerhardT, U., «Immanenz und Widerspruch. Die philosophischen Grundlagen der Soziologie Georg Simmels und ihr Verhältnis zur Lebensphilosophie Wilhelm Diltheys», en Zeitschrift für Philosophische Forschung, 25, 1971, pp. 276-292.

- "Georg Simmels Bedeutung für die Geschichte des Rollenbegriffs in der Soziologie», en Böhringer, H., y Gründer K. (eds.), Ästhetik und Soziologie um die jahrhundertwende: Georg Simmel, Frankfurt/M., 1976, 71-89.

Gerson, H., Die Entwicklung der ethischen Anschauung bei Georg Simmel, Diss, Berlín, 1932.

Grünewald, M., Die Philosophie Simmels mit besonderer Berücksichtigung ibrer Beziehung zum Pragmatismus, Diss, Breslau, 1925.

Hawthorn, H. B., "A Test of Simmel on the Secret Society. The Doukhobors of British Columbia», en American Journal of Sociology, núm. 62, 1956, pp. 1-7.

HazelrigG, L. E., "A Reexamination of Simmel's "The Secret and the Secret Society". Nine Propositions", en Social Forces, 47, 1969, pp. 323-330.

Heberle, R., "The Sociology of Georg Simmel. The Forms of Social Interaction", en Barnes, H. E. (ed.), Introduction to the history of sociology, Chicago, 1948, pp. 249-273.

Honigsheim, P., «Simmel», en Handwörterbuch der Sozialwissenschaften, tomo 9, Tübingen, 1956, pp. 270-272.

- "The Time and Thought of the Young Simmel», en Wolff, K. H. (ed.), Georg Simmel. 18581918. A Collection of Essays, with Translations and a Bibliography, Ohio State University, 1959, pp. 167-174.

- "A Note on Simmel's Anthropological Interests», en Wolff, K. H. (ed.), Georg Simmel. 18581918. A Collection of Essays, with Translations and a Bibliography, Ohio State University, 1959, pp. 175-179.

HÜBner-FunK, S., "Ästhetizismus und Soziologie bei Georg Simmel», en Böhringer, H., y Gründer, K. (eds.), Ästhetik und Soziologie um die jahrhundertwende: Georg Simmel, Frankfurt/M., 1976, pp. 44-70.

- Georg Simmels Konzeption von Gesellschaft. Ein Beitrag zum Verhältnis von Soziologie, Ästhetik und Politik, Köln, 1982.

JoËL, K., «Eine Zeitphilosophie», en Neue Deutsche Rundschau, 12, 1901, pp. 812-826.

Kaern, M.; Phillips, B. S., y Cohen, R. S. (eds.), Georg Simmel and Contemporary Sociology, Dordrecht/Londres/Boston, Kluwer Academic Publishers, 1990.

Kintzele, J., y Scheneider, P., Georg Simmels Philosophie des Geldes, Frankfurt am Main, 1993.

KIsS, G., Einführung in die soziologischen Theorien, tomo 2, Opladen, 1975.

KISTIAKOWSKI, Th., Gesellschaft und Einzelwesen. Eine methodologische Studie, Berlín, 1899.

Klemmt, A., Georg Simmel. Eine kritische Charakter-Studie und Erläuterung der Grundprobleme der gegenwärtigen Philosophie, Diss, Berlín.

Korgen, D., "Georg Simmel als Geldapologet», en Dokumente des Sozialismus, núm. 5, 1905, pp. 317-323.

- "Georg Simmels soziologischer Rationalismus», en Archiv für Sozialwissenschaft und Sozialpolitik, 31, 1910, pp. 908-924.

KöHnke, C. K., Der junge Simmel in Theoriebeziehung und sozialen Bewegungen, Frankfurt am Main, 1996.

KOnAU, E., Raum und soziales Handeln. Studien zu einer vernachlässigten Dimension soziologischer Theoriebildung, Stuttgart, 1977.

Kracauer, S., «Georg Simmel», en Logos, 9, 1920, pp. 307-338.

Landmann, M., «Konflikt und Tragödie. Zur Philosophie Georg Simmels», en Zeitschrift für philosophische Forschung, núm. 6, 1951, pp. 115-133. 
Landmann, M., «Bausteine zur Biographie», en Gassen, K., y Landmann, M. (eds.), Buch des Dankes an Georg Simmel. Briefe, Erinnerungen, Bibliographie, Berlín, 1958, pp. 11-30.

— «Georg Simmel als Prügelknabe», en Philosophische Rundschau, 14, 1967, pp. 258-274.

- «Einleitung des Herausgebers», en Simmel, G., Das individuelle Gesetz. Philosophische Exkurse, Frankfurt/M., 1968, pp. 7-29.

— «Georg Simmel. Konturen seines Denkens», en Böhringer, H., y Gründer, K. (eds.), Ästhetik und Soziologie um die Jahrhundertwende: Georg Simmel, Frankfurt/M., 1976, pp. 3-17.

LaWrence, P., "Introduction to the Life and Work of Georg Simmel», en G. Simmel. Sociologist and European, Sunbury - on - Thames, 1976, pp. 3-53.

LÉGer, F., La pensée de Georg Simmel. Contribution à l'histoire des idées en Allemagne au début du $X X^{e}$ siècle, tomo 3, Diss, París (O. J.).

LEVInE, D. N., "The Structure of Simmel's Social Thought», en Wolff, K. H. (ed.), Georg Simmel. 1858-1918. A Collection of Essays, with Translations, and a Bibliography, Ohio State University, 1959, pp. 9-32.

- «Some Key Problems in Simmel's Work», en Coser, L. A. (ed.), Georg Simmel, Englewood Cliffs, 1965, pp.97- 115.

— «Introduction», en Simmel, G., On Individuality and Social Forms, Chicago, IX-LXV, 1971.

- «Simmel at a Distance. On the History and Systematics of the Sociology of the Stranger», en Sociological Focus, 10, 1977, pp. 15-29.

- Simmel and Parsons. Two Approaches to the Study of Society, New York, 1980.

- "Sociology's Quest for the Classics. The Case of Simmel», en B. Rhea (ed.), The future of the Sociological classics, London, 1981, pp. 60-80.

Levine, D. N.; Carter, E. B., y Gorman, E. M., «Simmel's Influence on American Sociology», en American journal of Sociology, 81, 1976, pp. 813-845, pp. 1112-1132 (deutsche Übersetzung, en W. Lepenies [ed.], Geschichte der Soziologie, tomo 4, Frankfurt/M., 1981, pp. 32-81).

Lieber, H.-J., Kulturkritik und Lebensphilosophie. Studie zur deutschen Philosophie der Jahrhundertwende, Darmstadt, 1974.

Lieber, H.-J., y Furth, P., «Zur Dialektik der Simmelschen Konzeption einer formalen Soziologie», en Gassen, K., y Landmann, M. (eds.), Buch des Dankes an Georg Simmel. Briefe, Erinnerungen, Bibliographie, Berlín, 1958, pp. 39-59.

Liebeschütz, H., Von Georg Simmel zu Franz Rosenzweig. Studien zum jüdischen Denken im deutschen Kulturbereich, Tübingen, 1970.

LipmanN, M., «Some Aspects of Simmel's Conception of the Individual», en Wolff, K. H. (ed.), Georg Simmel. 1858-1918. A Collection of Essays, with Translations, and a Bibliograpby, Ohio State University, 1959, pp. 119-138.

LÜвBE, H., «Simmel», en Staatslexikon, tomo 7, Freiburg, 1962, pp. 80-82.

- Politische Philosophie in Deutschland, Munich, 1974.

LUKÁCS, G., «Über Georg Simmel», en Gassen, K., y Landmann, M. (eds.), Buch des Dankes an Georg Simmel. Briefe, Erinnerungen, Bibliographie, Berlín, 1958 (1918), pp. 171-176 .

- Die Zerstörung der Vernunft, 3, Darmstadt-Neuwied (I. Aufl. 1954), 1974.

Mamelet, A., "La philosophie de Georg Simmel», en Revue de Métaphysique et de Morale, núm. 20, 1912, pp. 567-612; 682-717; 825-877; núm. 21, 1913, pp. 390-435.

MASAryK, Th. G., "Simmels Soziologie», en Zeitschrift für Sozialwissenschaft, 12, 1909, pp. 600-607.

Maus, H., "Simmel in German Sociology», en Wolff, K. H. (ed.), Georg Simmel. 1858-1918. A Collection of Essays, with Translations, and a Bibliography, Ohio State University, 1959, pp. 180-200.

MAYNTZ, R., "Simmel», en Sills, D. L. (ed.), International Encyclopedia of the Social Sciences, tomo 14, New York, 1968, pp. 251-258.

McLemore, S. D., "Simmel's "Stranger". A critique of the concept», en Pacific Sociological Review, 13, 1970, pp. 86-94.

Mills, Th., «Some Hypotheses on Small Groups from Simmel», en American Journal of Sociology, 63, 1958, pp. 642-650. 
Mongardini, C., "Aspetti della sociologia di Georg Simmel», en Simmel, G., Il conflitto della cultura moderna et altri saggi, Roma, VIICXCVI, 1976.

Müller, H., Georg Simmel als Deuter und Fortbilder Kants, Diss, Leipzig, 1935.

NaEgele, K. D., "Attachment and Alienation. Complementary Aspects of the Work of Durkheim and Simmel», en American Journal of Sociology, 63, 1968, pp. 580-589.

Nedelmann, B., «Strukturprinzipien der Soziologischen Denkweise Georg Simmels», en Kölner Zeitschrift für Soziologie und Sozialpsychologie, 32, 1980, pp. 559-573.

Nisbet, R. A., Sociology as an Art Form, London, 1977.

NisSEN, J., "Vergesellschaftung als Einstellung. Eine Betrachtung zu Simmels Soziologie», en Annalen der Philosophie und Philosophischen Kritik, 5, 1925/1926, pp. 77-108.

Nobs, M., Der Einheitsgedanke in der Philosophie Georg Simmels, Diss, Bern, 1926.

NowaK, St., Wstep do wydania polskiego (Einleitung zur polnischen Übersetzung, en Simmel, G., Sociologia, Warszawa, IXXXXI), 1975.

OAKES, G., «Introduction», en Simmel, G., Essays on Interpretation in Social Science, Manchester, 1980, pp. 3-94.

- Georg Simmel: on Women, Sexuality and Love, University of Yale Press, Princeton, 1984.

O’Neill, J., "On Simmel's "Sociological Apriorities" ", en Psathas, G. (ed.), Phenomenological Sociology, Issues and Applications, New York, 1973, pp. 91-106.

Pohlmann, E., Das soziologisch-philosophische Werk Georg Simmels und sein geistesgeschichtliches Umfeld, Diss, Freiburg i. Br., 1979.

Rammstedt, O., Simmel und die frühen Soziologen. Nähe und Distanz zu Durkheim, Tönnies und Max Weber, Frankfurt am Main, 1988.

Rammstedt, O., y Watier, P. (eds.), Georg Simmel et les sciences humaines, París, Meridiens Klincksieck, 1992.

RaYL, (ed.), Formal Sociology: The Sociology of Georg Simmel, Cheltelham, Elgar, 1991.

SChmalenbach, H., "Simmel», en Sozialistische Monatshefte, 25, 1919, pp. 283-288.

SCHmidT, C., "Eine Philosophie des Geldes», en Sozialistische Monatshefte, 5, 1905, pp. 180-185.

SCHMOller, G., "Simmels Philosophie des Geldes», en Jahrbuch für Gesetzgebung, Verwaltung und Volkswirtschaft im Deutschen Reich, 25, 1901, pp. 799-816.

SCHNABEL, P. E., Die soziologische Gesamtkonzeption Georg Simmels. Eine wissenschaftshistorische und wissenschaftstheoretische Untersuchung, Stuttgart, 1974.

- «Georg Simmel», en Käsler, D. (ed.), Klassiker des soziologischen Denkens, tomo 1, Von Comte bis Durkheim, Munich, 1976, pp. 267-311.

SChrader-Klebert, K., «Der Begriff der Gesellschaft als regulative Idee. Zur transzendentalen Begründung der Soziologie bei Georg Simmel», en Soziale Welt, 19, 1968, pp. 97-118.

Simmel, H., "Auszüge aus den Lebenserinnerungen», en Böhringer, H., y Gründer, K. (eds.), Ästhetik und Soziologie um die jahrhundertwende: Georg Simmel, Frankfurt/M., 1976, pp. 247-268.

Sorokin, P., Contemporary Sociological Theories, New York, 1928.

SpanN, O., "Zur Kritik des Gesellschaftsbegriffes der modernen Soziologie», en Zeitschrift für die gesamte Staatswissenschaft, 61, 1905, pp. 302-344.

- "Wirtschaft und Gesellschaft. Eine dogmenkritische Untersuchung», en Gesamtausgabe, tomo 1 (= Frühe Schriften), Graz, 1974 (Erstausgábe 1907).

Spykman, N. J., The social Theory of Georg Simmel, New York, 1925.

Steinhoff, M., "Die Form als soziologische Grundkategorie bei Georg Simmel», en Kölner Vierteljahreshefte für Soziologie, 4, 1924/1925, pp. 215-259.

Susman, M., "Pole jüdischen Denkens. Hermann Cohen und Georg Simmel», en Die Tat, 15, 1923/1924, pp. 385-389.

- Die geistige Gestalt Georg Simmels, Tübingen, 1959.

TARTLER, R., "Georg Simmels Beitrag zur Integrations- und Konflikttheorie der Gesellschaft», en Jahrbuch für Sozialwissenschaft, 16, 1965, pp. 1-12.

Tenbruck, F. H., "Georg Simmel», en Kölner Zeitschrift für Soziologie und Sozialpsychologie, 10, 1958, pp. 587-614. 
Tenbruck, F. H., «Formal Sociology», en Wolff, K. H. (ed.), Georg Simmel. 1858-1918. A Collection of Essays, with Translations, and a Bibliography, Ohio State University, 1959, pp. 61-99.

TÖNNIES, F., «Simmel als Soziologe», en Frankfurter Zeitung, 9, 10, 1918.

TrNKA, H., "Inwieweit bedeuten Simmels "Philosophie des Geldes" und Molls "Logik des Geldes" eine Förderung der modernen Geldtheorie?», Diss. (Hochschule für Welthandel), Viena, 1948.

Troeltsch, H., "Zum Begriff und zur Methode der Soziologie», en Weltwirtschaftliches Archiv, 8, 1916, pp. 259-274.

Troeltsch, E., «Der Historismus und seine Probleme. I. Buch: Das logische Problem der Geschichtsphilosophie», en Gesammelte Schriften, III, Tübingen, 1922.

Turner, J. H., «Marx and Simmel Revisited. Reassessing the Foundations of Conflict Theory», en Social Forces, 53, 1975, pp. 618-627.

UlRICH, P. O., Immanenz und Transzendenz. Georg Simmels Entwurf einer nachchristlichen Religionsphilosophie, Frankfurt/M., 1981.

VIERKANDT, A., "Die Beziehung als Grundkategorie des sozialen Denkens», en Archiv für Rechtsund Wirtschaftsphilosophie, 9, 1915/1916, pp. 83-90; 214-225.

- «Programm einer formalen Gesellschaftslehre», en Kölner Vierteljahreshefte für Sozialwissenschaften, 1, 1921, pp. 56-66.

- «Simmel», en Encyclopedia of Social Sciences, tomo 14, 61, New York, 1934.

Wallisch-Prinz, B., A Sociology of Freedom. Georg Simmel's Theory of Modern Society, Diss, Bremen, 1977.

Walter, E. V., "Simmel's Sociology of Power. The Architecture of Politics», en Wolff, K. H. (ed.), Georg Simmel. 1858-1918. A Collection of Essays, with Translations, and a Bibliography, Ohio State University, 1959, pp. 139-166.

Watier, P. (ed.), La sociologie et l'experience du monde moderne, París, Meridiens Klincksieck, 1986.

Weber, M., "Georg Simmel as Sociologist, with an Introduction by D. N. Levine», en Social Research, 39, 1972, pp. 155-163.

Weingartner, R. H., Experience and Culture. The Philosophy of Georg Simmel, Middletown, Conn, 1960.

Wiese, L. von, "Soziologie als Einzelwissenschaft», en Schmollers Jahrbuch 44, 1920, pp. 347-367.

- "Zur Methodologie der Beziehungslehre», en Kölner Vierteljahreshefte für Sozialwissenschaften, 1, 1921, pp. 47-55.

WIESEHÖFER, W. P., Der unmetaphysische Mensch. Untersuchungen zur Anthropologie im Frühwerk Georg Simmels, Diss, Tübingen, 1975.

WolfF, K. H., "Introduction", en The sociology of Georg Simmel, traducido y editado por K. H. Wolff, Glencoe, Ill., XVII-LXIV, 1950.

- "The Challenge of Durkheim and Simmel», en American Journal of Sociology, 63, 1958, pp. 590-596.

- The Sociology of Georg Simmel, University of Chicago Press, Chicago, 1964.

WolfF, K. H. (ed.), Georg Simmel. 1858-1918. A Collection of Essays, with Translations, and a Bibliography, Ohio State University, 1959.

WoYslawSKI, H.-L., Georg Simmels Philosophie des kapitalistischen Geistes, Diss, Berlín, 1931. 


\section{TEXTO CLÁSICO}

